\title{
Promoção de iniciativas de arquivamento da web: um estudo a partir da rede de públicos estratégicos da UFRGS
}

\author{
Promotion of web archiving initiatives: a study based on UFRGS 'strategic public network \\ Marina Rodrigues Martins ${ }^{1}$, Moises Rockembach ${ }^{2}$ \\ ${ }^{1}$ Universidade do Vale do Rio dos Sinos: São Leopoldo, Rio Grande do Sul, Brasil. ORCID: https://orcid.org/0000-0002-6815-815X \\ 2 Universidade Federal do Rio Grande do Sul: Porto Alegre, Rio Grande do Sul, Brasil. ORCID: https://orcid.org/0000-0001-9057-0602
}

Autor para correspondência/Mail to: Marina Rodrigues Martins, mrodriguesmartins@gmail.com

Copyright (C) 2019 Martins \& Rockembach. Todo o conteúdo da Revista (incluindo-se instruções, política editorial e modelos) está sob uma licença Creative Commons Atribuição-NãoComercial-Compartilhalgual 3.0 Não Adaptada. Ao serem publicados por esta Revista, os artigos são de livre uso em ambientes educacionais, de pesquisa e não comerciais, com atribuição de autoria obrigatória. Mais informações em http://revistas.ufpr.br/atoz/about/submissions\#copyrightNotice.

\begin{abstract}
Resumo
Introdução: Descreve o fenômeno conhecido por arquivamento da web e o microambiente relacional de iniciativas implantadas pela Universidade de Columbia e pela Universidade de Harvard. Como problema central questiona como se con a potencial rede de públicos estratégicos da Universidade Federal do Rio Grande do Sul, visando a promover iniciativas de arquivamento da web no âmbito acadêmico, tendo em conta o ambiente relacional projetado a partir dos Órgãos da Administração Superior da Universidade e seu Programa de Pós-graduação em Comunicação. Método: É uma pesquisa exploratório-descritiva desenvolvida por meio de pesquisas bibliográfica, documental e análise de conteúdo. O entendimento sobre públicos ocorreu a partir dos enfoques da conceituação lógica, do poder e da comunicação. Resultados: $O$ estudo concluiu que os atores organizacionais exercem influência em diferentes níveis dentro das estruturas institucionais, cada um conforme suas responsabilidades, e que o sistema comunicacional denota papel importante para a efetividade das iniciativas. Alguns públicos mapeados agem na criação de coleções, como também podem vir a ser potenciais usuários dos arquivos web formados. Essas coleções são concebidas por várias organizações coletoras, não apenas as entidades macropromotoras das iniciativas. Quanto maior a quantidade de coleções arquivadas, maior a rede de públicos envolvidos na promoção da tecnologia. Conclusão: $O$ escopo de coleta das iniciativas varia conforme os interesses de cada organização coletora, apresentando coleções com diferentes classificações: institucional, regional, nacional, internacional, por fato e evento. Elas preservam tanto a história organizacional de suas entidades, como também promovem o ensino, a pesquisa e a extensão de suas comunidades.
\end{abstract}

Palavras-chave: Arquivamento da web; Arquivo web; Mapeamento de públicos; Relações Públicas; Perfil de públicos.

\begin{abstract}
Introduction: It describes the phenomenon known as web archiving and the relational microenvironment of initiatives implemented by Columbia University and Harvard University. As a central problem, it questions how the potential network of strategic audiences of the Federal University of Rio Grande do Sul is configured, aiming to promote web archiving initiatives in the academic scope, taking into account the relational environment projected from the Higher Administration Bodies of the University and its Communication Graduate Program. Methods: It is exploratory-descriptive research developed through bibliographic and documentary research, and content analysis. The understanding of audiences came from the approaches of logical conceptualization, power, and communication. Results: The study concluded that the organizational actors exert influence at different levels within the institutional structures, each according to their responsibilities and that the communication system shows an important role in the effectiveness of the initiatives. Some mapped audiences act in the creation of collections, but they can also become potential users of the formed web files. These collections are designed by various collecting organizations, not just the macro entities promoting the initiatives. The greater the number of archived collections, the greater the network of audiences involved in promoting the technology. Conclusion: The scope of the collection of the initiatives varies according to the interests of each collecting organization, presenting collections with different classifications: institutional, regional, national, international, by fact and event. They preserve both the organizational history of their entities, as well as promoting teaching, research, and the extension of their communities.
\end{abstract}

Keywords: Web archiving; Web archive; Mapping audience; Public Relations; Public profile.

\section{INTRODUÇÃO}

O arquivamento da web é o processo que envolve a seleção, a coleta, o armazenamento e a recuperação de websites com o objetivo de preservar os diversos conteúdos digitais presentes na World Wide Web (www). A captura destes materiais pode ser extensiva, arquivando maior quantidade de websites em um nível superficial, como também intensiva, capturando em menor escala, mas em maior profundidade de navegação (Masanès, 2006). A cobertura das capturas - Uniform Resource Locator (URLs) - domínios de interesse que devem ser capturados e preservados nos arquivos da web - classifica o perfil de conteúdo das coleções, que podem ser acadêmico-científica, institucional, nacional, por regiões/continentes, por fatos, entre outros.

Entre as organizações que desenvolvem a tecnologia no exterior estão entidades não governamentais, públicas e de ensino (Ferreira, Martins, \& Rockembach, 2018). Cada organização possui um ambiente que é influenciado pelo nicho de atuação (Henriques, [201-]) e demanda apoio dos diferentes grupos de influência. Por sua vez, as iniciativas de arquivamento da web devem estar alinhadas com a missão, a visão e as estratégias de atuação das organizações; essas últimas, para existirem e atuarem, dependem de pessoas e, por vezes, de outras organizações/grupos em seu âmbito interno e externo, estabelecendo os mais variados tipos de relacionamento. Esse corpo relacional se configura como públicos, também chamados aqui de grupos de influência. São agentes que 
influenciam na conquista da missão da organização e são influenciados por ela, uma vez que possuem interesses em comum (Simões, 2001).

Para garantir o sucesso dos projetos de arquivamento da web no ambiente complexo das organizações é fundamental estabelecer estratégias que englobam todo o processo, desde a seleção até o acesso dos conteúdos pelos usuários (Brügger, 2005). Nessa perspectiva o Web Archiving Life Cycle Model, exemplo orgânico desenvolvido pela equipe do serviço Archive-it, apresenta fases importantes para o processo de implantação em diferentes estruturas organizacionais. Dentre as que representam decisões de alto nível estão: clareza dos objetivos do programa; revisão e planejamento dos recursos disponíveis (fluxo de trabalho, finanças, experiência, pessoal, colaboradores e outros) e gerenciamento de riscos, ligado aos direitos autorais dos dados coletados (Bragg \& Hanna, 2013). O mapeamento de públicos estratégicos contribuirá principalmente para estas etapas.

Assim sendo, apresenta-se o contexto do arquivamento da web a partir de duas iniciativas universitárias nos Estados Unidos da América - da Universidade de Columbia e da Universidade de Harvard. A partir do levantamento, delineia-se a possível rede de públicos para promover uma iniciativa que beneficia o ensino, a pesquisa e a extensão dentro da estrutura organizacional da UFRGS, com foco no Programa de Pós-graduação em Comunicação (PPGCOM), da Faculdade de Biblioteconomia e Comunicação (FABICO).

\section{PÚBLICOS EM RELAÇÕES PÚBLICAS}

São variados os conceitos e as nomenclaturas utilizadas nas referências de Relações Públicas (RP). Por muito tempo, as definições foram apoiadas no critério geográfico/espacial que o objeto — público — tomava diante da organização, apresentando a divisão simplificada em três tipos: interno, misto e externo. Simões (2001) conceitua que públicos são agentes de influência (parceiros da organização, pessoas ou conjunto de pessoas, grupos ou demais organizações) cujos interesses são afetados e afetam as ações da organização na consecução de sua missão ou que buscam o controle das decisões e das ações desta. Variam conforme complexidade, tamanho, potencial de poder que podem exercer, com variada probabilidade de bloquear metas e objetivos. Estes públicos fazem parte de um sistema social sustentado por um processo de comunicação que possui a informação como matéria-prima e se configura em uma linha tênue de cooperação e conflito entre as partes, ocorrendo de modo não linear (Simões, 2001; Steffen, 2008).

A conceituação lógica dos públicos (França, 2012) ocorre a partir de três critérios de relacionamento. O primeiro é o de dependência jurídica/situacional da organização/iniciativa perante seus públicos a fim de viabilizar sua constituição, existência e permanência, mantendo sua produtividade. Nesta categoria estão os públicos: essenciais constitutivos e os essenciais não-constitutivos ou de sustentação — primários (de alto envolvimento) e secundários (de médio envolvimento). O segundo é o de participação em prol dos interesses e na promoção institucional da iniciativa. Nessa categoria estão os não-essenciais, que exercem as atividades-meio. O terceiro é de interferência que o público pode exercer sobre a organização/iniciativa. São grupos externos, que não a constituem e nem a mantém, mas podem exercer pressão sobre os seus interesses.

\section{CONFIGURAÇÕES ESTRUTURAIS ORGANIZACIONAIS DE UNIVERSIDADES FEDERAISNO BRASIL}

A forma de organização de uma instituição influencia em seus resultados. Se mal estruturada, pode gerar atritos, frustrações, aprofundar disputas irrelevantes e salientar deficiências de gestão (Drucker, 1997). É a estrutura organizacional que define a interligação dos grupos especializados e pode ser definida como resultado do processo de distribuição da autoridade exercida por seus atores (públicos). O sistema de comunicação perpassa toda a estrutura da organização; é por meio dele que o poder é exercido para a tomada de decisão (Maximiliano, 1985; Vasconcellos \& Hemsley, 1986).

Machado (1998) cita o modelo tradicional tipo comissão e colegiado, o qual adota uma administração plural para tomada de decisão. Essas comissões e colegiados são formados por membros internos da organização, provenientes de diferentes segmentos, departamentos e profissões (Cury, 1988). No caso de universidades federais de ensino superior no Brasil, esse tipo de estrutura se concretiza por meio dos conselhos universitários, de curadores e de ensino, pesquisa e extensão, como o exemplo da UFRGS (1995).

A complexa estrutura organizacional do modelo tradicional exercida por meio de comissão e colegiado retrata a administração plural, porém demanda articulação entre os diversos atores. Para tanto, identificar os públicos na classificação lógica — de acordo com seus graus de influência — pode estimular a integração entre eles e facilitar a atuação estratégica, amenizando a realidade de trabalho simultâneo e otimizando recursos. 


\section{PROCEDIMENTOS METODOLÓGICOS}

Tratou-se de uma pesquisa exploratório-descritiva voltada a mapear os públicos que dão origem às iniciativas de arquivamento da web implantadas pelos objetos explorados e visou projetar esta realidade na UFRGS. Para tanto, o processo investigativo ocorreu nas três etapas. A primeira buscou entender o contexto de arquivamento da $w e b$ - como ocorre o processo, iniciativas e organizações promotoras, propósitos, escopos e políticas. Para isso, foi realizado levantamento bibliográfico associando os temas de arquivamento da web, públicos e estruturas organizacionais nas bases Scopus, Web of Science, Google Scholar e SciELO Citation Index. A busca contemplou artigos publicados entre 2010 e 2017.

A segunda etapa consistiu na coleta de dados das universidades internacionais por meio da pesquisa documental - estatutos, regimentos e organogramas, como também da pesquisa de conteúdo - comunicações e mensagens dos websites. Nessa fase, fez-se a leitura flutuante e coleta de dados, respeitando as categorias de governança, estrutura, visão, missão e classificação. As fontes foram escolhidas a partir da regra de pertinência, uma vez que corresponderam aos objetivos do estudo, período e procedimentos de análise (Bardin, 2004; Fonseca Junior, 2008).

A partir do levantamento das informações de cada universidade internacional e suas iniciativas, elaboraram-se quadros de recorte, organizando os dados para identificar seus públicos e suas respectivas funções dentro da estrutura organizacional, para então formar os gráficos hierárquicos (redes de relacionamento). A categorização dos grupos de influência ocorreu a partir da "Identificação e Mapeamento dos Públicos" (IMP), de (França, 2012): listaram-se os públicos que se relacionavam com as iniciativas, como também faziam parte da estrutura organizacional conforme suas regras de governança; determinaram-se os tipos de relacionamento entre organização/iniciativa/públicos; definiu-se o objetivo destes relacionamentos, a partir de suas responsabilidades na estrutura organizacional; indicou-se o nível de envolvimento, a partir dos graus de dependência, participação e interferência; e assim categorizaram-se os públicos entre: essenciais, não-essenciais, redes de interferência. Os quadros de recorte também englobaram os seguintes indicadores - tipo de relacionamento: administrativo, classista, institucional, legal, negócios, operacional, parceria total, política, profissional, social; nível de envolvimento: frequente, permanente, ocasional, sazonal ou nenhum, que poderia ser também "não identificado"; critérios de relacionamento: I. grau de dependência jurídica ou situacional organização/públicos; II. maior ou menor grau de participação dos públicos nas ações da organização e III. grau de interferência que determinado público pode exercer sobre a organização e seus negócios.

A terceira etapa dissertou sobre a estrutura organizacional da UFRGS, utilizando como subsídio o "Estatuto e o Regimento Geral da Universidade (1995)", além dos conteúdos disponíveis no website da Universidade, com suas respectivas extensões do domínio "ufrgs.br". A partir da identificação da governança e da estrutura, ocorreu o delineamento dos públicos de acordo com os passos citados na IMP.

As análises utilizadas foram a documental e a de conteúdo, uma vez que a primeira buscou contextualizar as iniciativas e a segunda analisar as comunicações e mensagens dos objetos. Os dados coletados nas fontes foram reunidos, categorizados e organizados em um formato diferente do original, a fim de apresentar os resultados de pesquisa (Bardin, 2004; Moreira, 2008).

\section{RESULTADOS}

Apresentamos a seguir os dados das iniciativas de arquivamento da web da Universidade de Harvard e da Universidade de Columbia (EUA), bem como a projeção para a estrutura da UFRGS.

\section{A INICIATIVA DE ARQUIVAMENTO DA WEB DA UNIVERSIDADE DE HARVARD}

A iniciativa é viabilizada por meio da tecnologia disponibilizada pelo Archive-it, serviço de arquivamento da web oferecido pela organização Internet Archive, a qual possibilita que a Universidade autogerencie suas coleções, coletando, criando e preservando conteúdos digitais de interesse de seus públicos (Archive-it.org, 2014).

As nove organizações coletoras - 1) Center for the History of Medicine; 2) Harvard Business School; 3) Harvard Graduate School of Design; 4) Harvard Kennedy School of Government; 5) Harvard Law School; 6) Harvard University Archives; 7) Reischauer Institute of Japanese Studies, Harvard University; 8) Schlesinger Library e 9) Western Languages Division, Harvard - configuram um corpus de 69 coleções, formadas pela captura de 17.083 links de websites internos e externos ao ambiente digital da Universidade.

As capturas incluíam: textos, imagens e outros formatos multimídia, páginas da web interligadas, boletins informativos e blogs. Essa iniciativa é amparada por uma rede formada por 23 perfis de públicos e seus respectivos subgrupos. Os públicos mapeados fazem parte não apenas da iniciativa, como também da governança e da estrutura da Universidade como um todo. Estes grupos podem influenciar na tomada de decisão devido às responsabilidades administrativas, jurídicas e financeiras que possuem na estrutura da Universidade como, por 
exemplo, autorizar e viabilizar financeiramente a iniciativa dentro da instituição; estabelecer parcerias com as organizações coletoras (internas e externas); apoiar na coleta de URLs de interesse de seus grupos específicos; e também no uso e no reúso das informações arquivadas por parte de suas comunidades.

\section{A INICIATIVA DE ARQUIVAMENTO DA WEB DA UNIVERSIDADE DE COLUMBIA}

Esta iniciativa também é viabilizada por meio do Archive-it. Na página oficial (https://library.columbia.edu/locati ons/cuarchives/resources/webarchives.html) da Biblioteca da Universidade de Columbia, na seção arquivos web, consta texto informativo de como funciona a política de seleção dos domínios preservados. É possível identificar alguns dos públicos que dão origem à iniciativa e criam parte das coleções - funcionários administrativos, pesquisadores e alumnis (ex-alunos).

Ao explorar o serviço Archive-it, no campo de busca "Explore all archives", buscando pela palavra-chave "Columbia University", apareceram como resultados 2.879 websites capturados, subdivididos em 15 coleções. Como organizações coletoras: Columbia University Libraries, a Columbia University e a Columbia University, School of Professional Studies. Ao realizar a busca separadamente, utilizando o nome das organizações coletoras, os resultados foram diferentes. Quando a palavra-chave foi "Columbia University Libraries", apareceram nove coleções e 2.835 websites. Já com a palavra-chave "Columbia University, School of Professional Studies" obtiveramse três coleções e seis websites. Ao se buscar por "American Assembly - Columbia University", o resultado foi "ainda não há conteúdo público disponível".

A soma das buscas pelos nomes das organizações coletoras totalizou 12 coleções, o que é diferente do resultado da palavra-chave principal "Columbia University", que identificou 15 coleções. O mesmo ocorreu com a quantidade de websites capturados, pois identificou-se uma diferença de 38 URLs. Ao analisar as 15 coleções, percebeu-se que três delas estão indiretamente ligadas à palavra Columbia, indicando uma possível dependência de parceria operacional e administrativa com públicos. Porém, ao aprofundar a pesquisa, na variável "Descrição" constatou-se que somente uma coleção — Global Webcomics Web Archive — faz parte direta do ambiente relacional da Universidade.

Após o levantamento total, foram constatadas 12 coleções, formadas pela captura de 2.841 websites internos e externos ao ambiente digital da Universidade. As coleções preservam uma variedade de conteúdos, incluindo principalmente textos, imagens, páginas da web interligadas, grupos de notícias, boletins informativos e blogs. Grande parte se caracteriza pelo perfil institucional, porém alguns escopos de coleta abrangem conteúdo internacional.

A governança geral da Universidade está nas mãos do Senado Universitário e ocorre democraticamente. Esse Senado é formado por representantes de diferentes setores, incluindo administrativo, faculdades, estudantes, instituições filiadas, equipe da biblioteca profissional, pesquisa e alumnis. Como a Universidade de Harvard, a Columbia também é subdividida em vários campi, possuindo o total de 16 unidades, entre faculdades e escolas (Columbia University, 1959). A pesquisa identificou também outros departamentos e escritórios ligados à Columbia University Libraries, os quais podem influenciar na tomada de decisão referente à iniciativa, uma vez que é a Biblioteca a promotora do projeto.

À vista disso, a iniciativa forma um total de 10 perfis de públicos, os quais dão origem às coleções e sustentam a iniciativa. Estes grupos influenciam em diferentes etapas do processo de arquivamento da web como, por exemplo, na operação e na manutenção de curadoria de coleções; na liberação de acesso aos conteúdos; na seleção/organização dos websites capturados e na manutenção do contrato de serviço fornecido pela organização Internet Archive.

\section{A POTENCIAL REDE DE PÚBLICOS DA UFRGS - ÓRGÃOS DA ADMINISTRAÇÃO SUPE- RIOR, FABICO E PPGCOM.}

Com sede em Porto Alegre, a UFRGS oferece cursos em diferentes áreas do conhecimento desde o ensino fundamental até a pós-graduação. Na estrutura são registrados mais de 700 grupos de pesquisa, formados por alunos de graduação e pós-graduação, técnicos de laboratório, docentes e visitantes, com um corpo de, aproximadamente, 14 mil pessoas (UFRGS, 2018a).

Conforme o Art. $7^{\circ}$ do Estatuto, para atingir seus objetivos a Universidade se estrutura em órgãos da administração superior e 29 unidades universitárias (Institutos centrais, faculdades ou escolas, com seus órgãos auxiliares), entre outros (Hospital universitário; institutos especializados e centros de estudos interdisciplinares). São órgãos da administração superior, segundo o Art. 9 , o Conselho de Curadores (CONCUR); o Conselho Universitário (CONSUN); o Conselho de Ensino, Pesquisa e Extensão (CEPE) e a Reitoria. Esses conselhos são geridos democraticamente e compostos pelas diferentes representações da comunidade universitária e outros influenciadores (docentes, discentes, ex-alunos, servidores técnico-administrativos, representantes de entidades de trabalhadores, culturais, como também do setor de ciência e tecnologia, além de membros externos, indicados 
pelo Ministério da Educação). Cada uma das 29 unidades e alguns dos 700 grupos de pesquisa possuem seus websites próprios com informações referentes a currículos; processos seletivos; bolsas; publicações; blogs; pesquisas; imagens; entre outras. Esses conteúdos são gerenciados pelas equipes internas de cada departamento e grupo, conforme seus interesses e de seus públicos (UFRGS, 2018b).

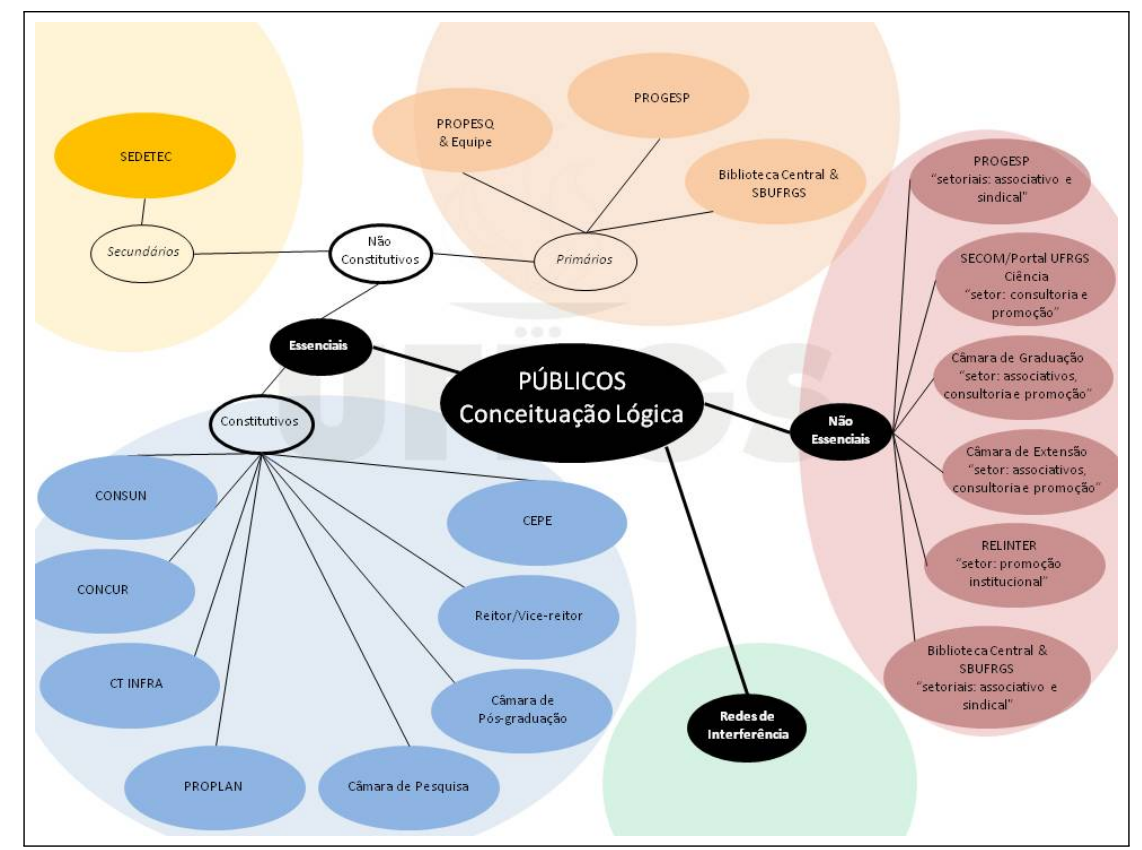

Figura 1. Gráfico hierárquico Órgãos da Administração Superior

Fonte: Dados da pesquisa, elaborado pelos autores (2019).

A Figura acima demonstra a rede composta por 16 públicos estratégicos ligados à Administração Superior da UFRGS. Considera-se que um mesmo público pode se encaixar em classificação diferente, devido a sua característica particular de tipo de relacionamento.

Vinculadas à Reitoria, estão diferentes Pró-Reitorias, como a Pró-Reitoria de Gestão de Pessoas (PROGESP), a Pró-Reitoria de Planejamento (PROPLAN) e a Secretaria de Desenvolvimento Tecnológico (SEDETEC), que podem contribuir para a implantação de um projeto de arquivamento da web na UFRGS, uma vez que, conforme a ordem citada, a partir de suas ações e responsabilidades é possível: a) conseguir apoio técnico-administrativo para operação e concretização da iniciativa, caso seja necessário alocação de servidores para execução e manutenção das atividades de arquivamento da $w e b$; b) buscar assistência desse público para as atividades da iniciativa no que tange a orçamentos, celebração e acompanhamento de contratos e convênios, aquisições de bens e serviços, importação de equipamentos e serviços para o suporte à pesquisa e c) promover a iniciativa para que haja parceria e colaboração, a fim de propiciar desenvolvimento tecnológico para a realização do arquivamento.

A FABICO é uma das 29 unidades universitárias, a qual oferece seis cursos de graduação e três programas de pós-graduação, sendo um em Comunicação (PPGCOM), um em Museologia e Patrimônio (PPGMUSPA) e um em Ciência da Informação (PPGCIN). A faculdade possui URL próprio com extensões específicas para cada curso de graduação, já os Programas de Pós-graduação (PPGs) possuem, além das extensões, seus endereços próprios para comunicação de informações (matrículas, disciplinas, quadro de docentes, publicações, linhas, projetos e grupos de pesquisa, documentos, formulários, entre outros) com seus públicos ((alias?), 2018). Cada curso stricto sensu possui um conselho, coordenado por uma comissão de pós-graduação com mandato de dois anos. Ambos, conforme os artigos $49^{\circ}$ e $50^{\circ}$ do Estatuto e Regimento Geral, são geridos democraticamente por docentes e discentes, tendo um coordenador com funções executivas (UFRGS,1995). Somente o PPGCOM possui 15 grupos de pesquisa devidamente registrados no Conselho Nacional de Desenvolvimento Científico e Tecnológico (CNPq).

Os 15 públicos identificados exercem diferentes níveis de poder e influência conforme suas funções na Unidade como, por exemplo, a criação, a extinção ou a reestruturação de departamentos; a aprovação dos planos de ação, a elaboração de relatórios de atividades e da proposta orçamentária; como também funde e cria comissões, assessorias ou mecanismos necessários ao cumprimento das atribuições da unidade acadêmica.

De modo geral, os grupos são variados, conforme complexidade, tamanho e potencial de poder que podem exercer nos quesitos de consulta, de decisão, de comportamento e de opinião. 


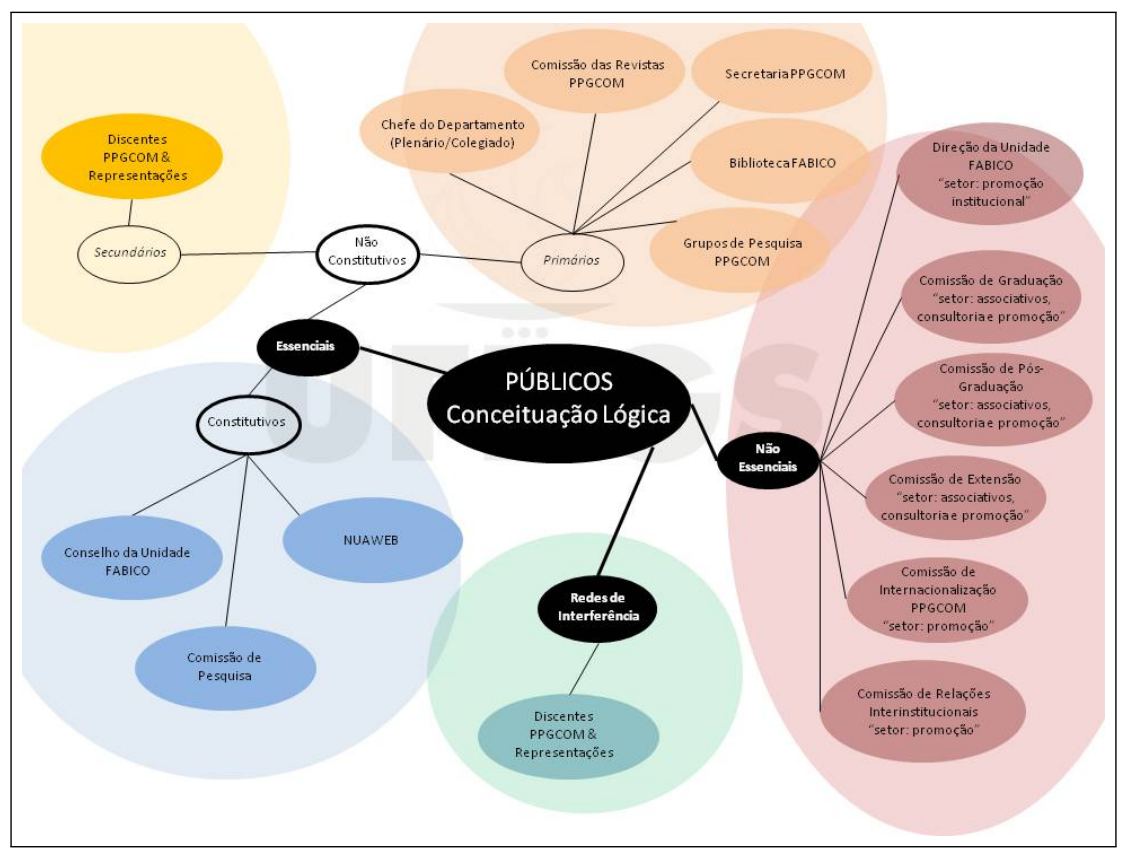

Figura 2. Gráfico hierárquico FABICO

Fonte: Dados da pesquisa, elaborado pelos autores (2019).

\section{CONSIDERAÇÕES FINAIS}

São diversos os perfis das organizações que utilizam a tecnologia de arquivamento da web internacionalmente, apresentando diferentes escopos de coleta (organizacionais, institucionais, de fatos e eventos específicos), conforme políticas e estratégias estabelecidas por suas governanças.

À vista das iniciativas internacionais observadas, alguns públicos mapeados agem na criação das coleções, como também podem vir a ser potenciais usuários dos arquivos web formados. Essas coleções são concebidas por várias organizações coletoras que não apenas as entidades macropromotoras. Algumas das organizações coletoras são partes das estruturas organizacionais das universidades, outras não. Por vezes, monitoram e capturam URLs externos - além dos domínios "columbia.edu" e "harvard.edu". Mesmo sendo viabilizadas pelo serviço Archive.it, pode-se dizer que são responsáveis pela política de arquivamento e dos conteúdos selecionados, porém não fica claro como e se exercem algum poder sobre a tecnologia.

Os três objetos de estudo são instituições de ensino superior, estruturadas física e organizacionalmente em vários campi universitários, oferecendo formações em múltiplas áreas de conhecimento. Suas estruturas organizacionais são interligadas por grupos especializados e podem ser definidas como resultado do processo de distribuição da autoridade exercida por seus públicos. O sistema comunicacional perpassa toda essa estrutura, possibilitando o exercício do poder em diferentes níveis. Sendo assim, percebe-se que os públicos, se categorizados logicamente a partir de suas funções e responsabilidades, podem facilitar as tomadas de decisão, principalmente no que tange às deliberações de alto nível do Web Archiving Life Cycle Model. Quanto maior a quantidade de coleções arquivadas, maior a rede de públicos envolvidos.

Para finalizar, conclui-se que, por meio da UFRGS e suas parcerias, é possível promover uma iniciativa de arquivamento da web no âmbito acadêmico e institucional desde que, a partir da conceituação lógica dos públicos, seja elaborado um planejamento estratégico de comunicação estimulando suas participações em prol da iniciativa. Isso se deve à característica multidisciplinar de seus pesquisadores. Para tanto, será necessário ter a percepção do sistema social para entender essas relações com os enfoques de comunicação e poder, a fim de potencializar coligações de apoio diante do ambiente e das relações políticas complexas que se fazem no contexto democrático da estrutura organizacional da UFRGS. 


\section{REFERÊNCIAS}

Archive-it.org. (2014). Website. Recuperado de https:// archive-it.org/.

Bardin, L. (2004). Análise de conteúdo (3a. ed.). Lisboa: Edições 70.

Bragg, M., \& Hanna, K. (2013). The web archiving life cycle model. Archive-It. Recuperado de https://archive-it.org/ static/files/archiveit_life_cycle_model.pdf

Brügger, N. (2005). Archiving websites. General Considerations and Strategies. Aarhus: Centre for Internet Research.

Columbia University. (1959). Charters and statutes. The Charters and Statutes are maintained by The Office of the Secretary . Recuperado de https://secretary.columbia.edu/ files/secretary/university charters and statutes/ UniversityStatues_December2017.pdf.

Cury, A. (1988). Organização e métodos: perspectiva comportamental $\mathscr{E}$ abordagem contigencial (4 ed). São Paulo: Atlas.

Drucker, P. F. (1997). Introdução à administração. São Paulo: Pioneira.

FABICO. (2018). Website. Universidade Federal do Rio Grande do Sul. Recuperado de http://www.ufrgs.br/fabico (19 de novembro de 2018)

Ferreira, L. B., Martins, M. R., \& Rockembach, M. (2018). Usos do arquivamento da web na comunicação científica. Prisma.com(36), 78-98. doi: 10.21747/16463153/36a5

Fonseca Junior, W. C. (2008). Análise de Conteúdo. In J. Duarte \& A. Barros (Eds.), Métodos e Técnicas de Pesquisa em Comunicação. São Paulo: Atlas.

França, F. (2012). Públicos: como identificá-los em nova visão estratégica: business relationship (3a. ed.). Ed. Yendis.

Henriques, Z. S. ([201-]). Ambiente empresarial e competitividade: a ligação da estratégia à execução. Recuperado de http://www.unimep.br/phpg/mostraacademica/anais/ $4 \mathrm{mostra} / \mathrm{pdfs} / 572 . \mathrm{pdf}$

Machado, N. d. S. (1998). Configurações estruturais em organizações universitárias: o caso a universidade do oeste de santa catarina (Dissertação de mestrado). Universidade Federal de Santa Catarina, Brasil.

Masanès, J. (2006). Web archiving g. Paris, FRA: SpringerVerlag Berlin Heidelberg.

Maximiliano, A. C. A. (1985). Introdução à administração (2 ed). São Paulo: Atlas.

Moreira, S. (2008). Análise documental como método e como técnica. In Duarte, j. \& barros, a. (orgs.) (v. 2, p. 269-279). São Paulo: Atlas.

PROGESP. (2018). Website. Universidade Federal do Rio Grande do Sul. Recuperado de http://www.ufrgs.br/ progesp/progesp-1 (20 de novembro de 2018)

PROPLAN. (2018). Website. Universidade Federal do Rio Grande do Sul. Recuperado de https://www.ufrgs.br/ proplan/quem-somos/apresentacao/ (21 de novembro de 2018)

SEDETEC. (2018). Website. Universidade Federal do Rio Grande do Sul. Recuperado de https://www.ufrgs.br/ sedetec/ (22 de novembro de 2018)

Simões, R. P. (2001). Relações públicas e micropolítica. São Paulo: Summus.
Steffen, A. M. (2008). O conceito de público em relações públicas. Porto Alegre: Cidadela.

Universidade Federal do Rio Grande do Sul. (1995). Estatuto e regimento geral da Universidade Federal do Rio Grande do Sul. Recuperado de https:// www.ufrgs.br/institutodeartes/wp-content/uploads/2018/ 03/Estatuto-e-Regimento-Geral-da-UFRGS.pdf

Universidade Federal do Rio Grande do Sul. (2018a). Pesquisa e Inovação. Recuperado de http://www.ufrgs.br/ ufrgs/pesquisa-e-inovacao/apresentacao (19 de novembro de 2018)

Universidade Federal do Rio Grande do Sul. (2018b). Unidades Regionais e Acadêmicas. Recuperado de http:// www.ufrgs.br/ufrgs/a-ufrgs/unidades-academicas (22 de novembro de 2018)

Vasconcellos, E., \& Hemsley, J. R. (1986). Estrutura das organizações: estruturas tradicionais, estruturas para inovação, estrutura matricial. São Paulo: Pioneira.
Martins, M. R. \& Rockembach, M. (2019). Promoção de iniciativas de arquivamento da web: um estudo a partir da rede de públicos estratégicos da UFRGS. AtoZ: novas práticas em informação e conhecimento, 8(2), 99 - 105. Recuperado de: http://dx.doi.org/10.5380/ atoz.v8i2.71333 